\title{
frontiers
}

\section{Detection of Antibody Responses against SARS-CoV-2 in Plasma and Saliva from Vaccinated and Infected Individuals}

1 Jéromine Klingler ${ }^{1,2}$, Gregory S. Lambert ${ }^{1}$, Vincenza Itri ${ }^{1}$, Sean Liu ${ }^{1}$, Juan C. Bandres ${ }^{1,2}$,

2 Gospel Enyindah-Asonye ${ }^{1}$, Xiaomei Liu ${ }^{1,2}$, Viviana Simon ${ }^{1,3,5,6}$, Charles R. Gleason ${ }^{3}$, Giulio

3 Kleiner $^{3}$, Hsin-Ping Chiu ${ }^{3}$, Chuan-Tien Hung ${ }^{3}$, Shreyas Kowdle ${ }^{3}$, Fatima Amanat ${ }^{3,4}$, Benhur

4 Lee $^{3}$, Susan Zolla-Pazner ${ }^{1,3}$, Chitra Upadhyay ${ }^{1}$, Catarina E. Hioe e,2,3* $^{*}$

$5{ }^{1}$ Division of Infectious Diseases, Department of Medicine, Icahn School of Medicine at Mount Sinai,

6 New York, NY, USA

$7 \quad{ }^{2}$ James J. Peters VA Medical Center, Bronx, NY, USA

$8 \quad{ }^{3}$ Department of Microbiology, Icahn School of Medicine at Mount Sinai, New York, NY, USA

$9{ }^{4}$ Graduate School of Biomedical Sciences, Icahn School of Medicine at Mount Sinai, New York, NY, 10 USA

$11{ }^{5}$ Department of Pathology, Molecular and Cell Based Medicine Icahn School of Medicine at Mount

12 Sinai, New York, NY, USA

$13{ }^{6}$ Global Health and Emerging Pathogen Institute, Icahn School of Medicine at Mount Sinai, New

14 York, NY, USA

15 *Correspondence:

16 catarina.hioe@mssm.edu, catarina.hioe@va.gov

17 Keywords: SARS-CoV-2, COVID-19, vaccination, antibody isotypes, neutralization, ADCP, 18 complement fixation, saliva 
4121 words, 6 figures, 2 supplemental tables, 6 supplemental figures

\section{Abstract}

Antibodies (Abs) are essential for the host immune response against SARS-CoV-2, and all the vaccines developed so far have been designed to induce Abs targeting the SARS-CoV-2 spike. Many studies have examined $\mathrm{Ab}$ responses in the blood from vaccinated and infected individuals. However, since SARS-CoV-2 is a respiratory virus, it is also critical to understand the mucosal Ab responses at the sites of initial virus exposure. Here, we examined plasma versus saliva Ab responses in vaccinated and convalescent patients. Although saliva levels were significantly lower, a strong correlation was observed between plasma and saliva total Ig levels against all SARS-CoV-2 antigens tested. Virus-specific IgG1 responses predominated in both saliva and plasma, while a lower prevalence of $\operatorname{IgM}$ and IgA1 Abs was observed in saliva. Antiviral activities of plasma Abs were also studied. Neutralization titers against the initial WA1 (D614G), B.1.1.7 (alpha) and B.1.617.2 (delta) strains were similar but lower against the B.1.351 (beta) strain. Spike-specific antibody-dependent cellular phagocytosis (ADCP) activities were also detected and the levels correlated with spikebinding Ig titers. Interestingly, while neutralization and ADCP potencies of vaccinated and convalescent groups were comparable, enhanced complement deposition to spike-specific Abs was noted in vaccinated versus convalescent groups and corresponded with higher levels of IgG1 plus IgG3 among the vaccinated individuals. Altogether, this study demonstrates the detection of $\mathrm{Ab}$ responses after vaccination or infection in plasma and saliva that correlate significantly, although Ig isotypic differences were noted. The induced plasma Abs displayed Fab-mediated and Fc-dependent functions with comparable neutralization and ADCP potencies, but a greater capacity to activate complement was elicited upon vaccination. 


\section{Introduction}

44

45

46

47

48

49

50

51

52

53

54

55

56

57

58

59

60

61

62

63

64

65

66

67

68

69

70

71

72

73

74

75

76

77

78

79

80

81

82

83

84

85

86

87

88

Antibodies (Abs) are an essential component of the immune responses against coronavirus disease-2019 (COVID-19). In the USA, three COVID-19 vaccines have received an authorization for emergency use from the FDA: two messenger RNA (mRNA) vaccines from Pfizer-BioNTech (BNT162b2) and Moderna (mRNA-1273), and one adenovirus-vectored vaccine from Johnson \& Johnson/Janssen (Ad26.CoV2.S). All three vaccines are designed to induce Abs targeting SARSCoV-2 spike $(1,2)$, a membrane-anchored protein on the viral surface that contains the receptorbinding domain (RBD) necessary for binding and entry into the host cells (3-5). Other vaccines utilized in other countries also function to generate Abs against SARS-CoV-2 spike protein (6). In addition, several monoclonal Abs targeting spike protein are under development (7), and three have been authorized for emergency use by the FDA for the treatment of mild to moderate nonhospitalized COVID-19 patients: REGEN-COV (Casirivimab with Imdevimab), Eli Lilly (Bamlanivimab and Etesevimab) and Vir Biotechnology/GlaxoSmithKline (Sotrovimab).

Many studies have evaluated $\mathrm{Ab}$ responses against SARS-CoV-2 elicited by infection or vaccination, but most examined Abs in the blood. Considering that SARS-CoV-2 is a respiratory virus, Abs in the mucosal sites would serve as the frontline defense against this virus; however, limited data are currently available. Similarities and differences have been noted in the distribution of Ig isotypes in the blood and mucosal tissues. The primary Abs found in the blood are IgG, representing $\sim 75 \%$ of serum Ig. Among the four IgG subtypes, IgG1 and IgG2 comprise $66 \%$ and $23 \%$ of IgG, whereas IgG3 and IgG4 are minor components ( $<10 \%$ each). IgM and IgA are also abundant in blood and constitute $10 \%$ to $15 \%$ of serum Ig. IgA is the major Ab isotype of the mucosal immune system and exists as IgA1 and IgA2 (8). Of these two subtypes, IgA1 Abs predominate in both serum and secretions, but IgA2 percentages are higher in secretions than in serum. Consistent with this information, our previous study demonstrated that anti-spike $\mathrm{Ab}$ responses in convalescent plasma collected 1-2 months post-infection, were dominated by IgG1, although the levels varied tremendously among subjects (12). Variable levels of $\operatorname{IgM}$ and $\operatorname{IgA1}$ were also detected and constituted the prominent Ig isotypes in some individuals. Other studies have shown that SARS-CoV-2-specific IgG, IgM and IgA responses could be detected in serum and saliva from COVID-19 patients, even though IgM and IgA declined more rapidly (9-11). However, the isotypes of vaccine-elicited $\mathrm{Ab}$ responses in mucosa have not been studied so far.

While the primary antiviral function of Abs is to neutralize virions, Abs also have nonneutralizing effector functions mediated via their Fc fragments. Virus-neutralizing activity was detected in IgG, IgM, and IgA fractions from COVID-19 convalescent plasma (12). COVID-19 vaccines also demonstrate the capacity to elicit potent neutralizing Ab responses (13-17). However, the full properties of Abs elicited by vaccination or infection are not yet known. In particular, limited data exists for Fc-mediated activities induced by vaccination which could play a role in vaccine efficacy (18). The binding of anti-spike Abs to virions, infected cells, or soluble spike proteins creates immune complexes capable of engaging Fc receptors (FcRs) or complement via the Abs' Fc fragments $(19,20)$. These interactions are determined by the Ig isotypes, as each isotype engages distinct FcRs and activates the complement system with varying potency $(19,21)$. The FcR engagement triggers a cascade of intracellular signals critical for Fc-mediated activities, including $\mathrm{Ab}$-dependent cellular phagocytosis (ADCP) and Ab-dependent cellular cytotoxicity (ADCC). Binding of $\mathrm{Clq}$, the first component in the classical complement pathway, to Fc fragments on immune complexes activates the downstream complement cascade, resulting in the deposition of $\mathrm{C} 3$ and C4 degradation products. Depositions initiates the generation of C5 convertase and the assembly of the membrane-attack complex which is responsible for complement-mediated lysis. Complement- 
medRxiv preprint doi: https://doi.org/10.1101/2021.05.11.21256972; this version posted December 7, 2021. The copyright holder for this preprint (which was not certified by peer review) is the author/funder, who has granted medRxiv a license to display the preprint in perpetuity.

It is made available under a CC-BY-NC SARRS-

89

90

91

92

93

94

95

96

97

98

99

100

101

102

103

104

105

106

107

108

109

110

111

112

113

114

115

116

117

118

119

120

121

122

123

124

125

126

127

128

129

130

131

132

133

opsonized immune complexes also interact with complement receptors on leukocytes to trigger effector functions, including complement-dependent cell-mediated phagocytosis and cytotoxicity $(22,23)$.

In this study, we assessed $\mathrm{Ab}$ responses elicited against different SARS-CoV-2 antigens from plasma and saliva samples collected from both vaccinated and convalescent donors using a multiplex bead assay that was developed in our previous study $(12,24)$. Saliva was used as a model for oral and upper respiratory mucosal secretions, and both saliva and blood specimens from each donor were obtained simultaneously. We further compared spike- and RBD-specific Ig isotypes in the same pairs of plasma and saliva samples. Additionally, vaccine- and infection-induced plasma Abs were examined for virus neutralization and Fc-dependent functions that included ADCP, C1q binding and C3d deposition. This study provides evidence for distinct SARS-CoV-2-specific Ig isotypes in plasma compared to saliva and differences in complement binding activities associated with Ig isotype profiles.

\section{Methods}

Recombinant proteins. SARS-CoV-2 spike and RBD proteins were produced as described $(25,26)$. S1, S2, and nucleoprotein antigens were purchased from ProSci Inc, CA (\#97-087, \#97-079 and \#97-085, respectively).

Human specimens. Plasma and saliva specimens were obtained from volunteers enrolled in Institutional Review Board-approved protocols at the Icahn School of Medicine at Mount Sinai (IRB\#17-00060, IRB\#19-01243) and the James J. Peter Veterans Affairs Medical Center (IRB\#BAN-1604): RN\#1, RN\#4 and RV\#1-5 after immunization; RP\#2-5, 7, 12, 13 after infection; and four contemporaneous non-vaccinated COVID-19-negative subjects. Thirteen additional convalescent plasma samples (CVAP samples) were obtained from 134-229 days after symptom onset under the "Evaluation of the clinical significance of two COVID-19 serologic assays" project, which received ethical approval from the James J Peters Veterans Affairs Medical Center Quality Improvement committee. Post-immunization plasma were also collected from 20 participants in the longitudinal observational "Protection Associated with Rapid Immunity to SARS-CoV-2" (PARIS) study, which was approved by the Icahn School of Medicine at Mount Sinai Institutional Review Board (IRB\#20-03374). The clinical data are summarized in Supplementary Tables 1 and 2. All participants signed written consent forms prior to sample and data collection. All participants provided permission for sample banking and sharing. All samples were heat-inactivated before use.

Multiplex bead Ab binding assay. Measurement of total Ig and Ig isotypes to SARS-CoV-2 antigen-coupled beads was performed as described (12). The quantification was based on MFI values at the designated sample dilutions. For total Ig responses, specimens were diluted 4-fold from 1:100 to $1: 6,400$ or 102,400 (plasma) or 2-fold from 1:2 to 1:16 (saliva), reacted with antigen-coated beads, and treated sequentially with biotinylated anti-human total Ig antibodies and PE-streptavidin. Titration curves were plotted for each antigens tested and the end-point titers were determined. The isotyping assays were performed at one dilution (1:200 for plasma, 1:4 for saliva) using human Ig isotype or subclasses antibodies and the MFI values were shown. Complement deposition onto plasma Abs reactive with spike and RBD were measured according to (27) with modifications. For the C1q assay, beads with spike-Ab or RBD-Ab complexes were incubated with C1q Component from Human Serum (Sigma, \#C1740) for 1 hour at room temperature, followed by an anti-C1q-PE antibody (Santa Cruz, \#sc-53544 PE). For the C3d assay, Complement Sera Human (33.3\%, Sigma, \#S1764) was added to the beads for 1 hour at $37^{\circ} \mathrm{C}$, followed by a biotinylated monoclonal anti-C3d antibody (Quidel, \#A702). The relative levels of C1q and C3d deposition were obtained as MFI, from

This is a provisional file, not the final typeset article 
which titration curves were plotted and areas-under the curves (AUC) were calculated.

Virus neutralization. Recombinant SARS-CoV-2 viruses encoding GFP and bearing SARSCoV-2 spike proteins of the initial WA1 strain (D614G, designated WT), B.1.1.7 (alpha), B.1.351 (beta) or B.1.617.2 (delta) variants were used in neutralization assays as described $(12,28,29)$. Virus infection in 293T-hACE2-TMPRSS2 cells initially seeded on collagen-coated 96-well plates was detected by $\mathrm{GFP}^{+}$cells. At 18-22 hours post infection, GFP counts were acquired by the Celigo imaging cytometer (Nexcelom Biosciences, version 4.1.3.0). Each condition was tested in duplicate.

ADCP. ADCP assays were performed using a reported protocol (30) with some modifications. FluoSpheres carboxylate-modified microspheres (Thermo Fisher, \#F8823) were coupled with SARS-CoV-2 spike protein using the xMAP Antibody Coupling Kit (5 $\mu \mathrm{g}$ protein $/ 36.4 \times 10^{9}$ beads, Luminex \#40-50016). Spike-conjugated microspheres were incubated with diluted plasma for 2 hours at $37^{\circ} \mathrm{C}$ in the dark. After washing and centrifugation $(2,000 \mathrm{~g}, 10$ minutes $)$, the beads $\left(\sim 3 \times 10^{8}\right.$ beads, $10 \mu \mathrm{L} /$ well $)$ were incubated with THP- 1 cells $\left(0.25 \times 10^{5}\right.$ cells, 200 $\mu \mathrm{L} /$ well) for 16 hours. The samples were analyzed on an Attune NxT flow cytometer (Thermo Fisher, \#A24858). Data analysis was performed using FCS Express 7 Research Edition (De Novo Software).

Statistical analysis. Statistical analyses were performed as designated in the figure legends using GraphPad Prism 8 (GraphPad Software, San Diego, CA).

\section{Results}

Detection of plasma and saliva Ab responses to SARS-CoV-2 antigens after vaccination and infection. Paired plasma and saliva specimens were collected from seven healthy recipients of COVID-19 mRNA vaccines and seven convalescent COVID-19 patients. Among vaccinees, two individuals received the Pfizer-BioNTech vaccine and five received the Moderna vaccine. Samples were collected 15-37 days after the second vaccine dose (Supplemental Table 1A). Convalescent patients presented with varying disease severity and donated samples 189-256 days post symptom onset (Supplemental Table 1B). In addition, samples from four COVID-19-negative non-vaccinated donors were tested in parallel and used to establish cut-off values.

Plasma and saliva samples were titrated for total Ig against SARS-CoV-2 spike, RBD, S1, S2, and nucleoprotein antigens. Bovine serum albumin (BSA) served as a negative control. Titration curves were ploted (Supplemental Figure 1), and the end-point titers were calculated (Figure 1A). All plasma specimens from vaccinated subjects exhibited titrating amounts of Ig against spike, RBD, $\mathrm{S} 1$ and $\mathrm{S} 2$ above the cut-off levels, although $\mathrm{S} 2$ reactivity was notably lower. As expected, reactivity was not observed against nucleoprotein, apart from one sample that showed weak reactivity slightly above the cut-off value. On the other hand, convalescent plasma samples displayed titrating Ig against spike, RBD, S1, S2 as well as nucleoprotein, and S2 reactivity was again the weakest. The presence of nucleoprotein-specific Abs differentiated convalescent from vaccinated subjects; these antibodies were present in plasma from convalescent but not vaccinated subjects (Figure 1A, Supplemental Figure 1). Correlation analyses further indicated that the levels of Abs against spike and each of spike fragments (RBD, S1, S2) correlated well in both groups but no correlation was found between spike and nucleoprotein Ab levels (Supplemental Figure 2). A similar pattern of reactivity and correlation was seen with saliva samples (Supplemental Figure 1, Supplemental Figure 2), albeit saliva titers were about 3 log lower compared to plasma titers (Figure 1A). 
We then calculated the number of responders (i.e. number of individuals reaching levels above cut-offs) and found that $100 \%$ of vaccinated and convalescent subjects showed plasma Ig reactivity to spike, $\mathrm{RBD}, \mathrm{S} 1$, and $\mathrm{S} 2$, and all convalescent subjects displayed plasma reactivity to nucleoprotein (Figure 1B). By contrast, only some vaccinated and convalescent subjects had positive saliva Ig reactivity. Five of the 7 saliva specimens from the vaccinated group exhibited Ig reactivity against RBD and 2 of the 7 against S2. Depending on the antigens, saliva Ig reactivity was also detected in one to six of the seven convalescent individuals. Nonetheless, the levels of total $\mathrm{Ig}$ in plasma and saliva correlated significantly for spike, S1, and other tested antigens (Figure 1C and data not shown).

Similarities and differences in Ig isotypes against SARS-CoV-2 spike and RBD present in plasma versus saliva from vaccinated and convalescent subjects. The plasma and saliva specimens were subsequently evaluated for total $\operatorname{Ig}, \operatorname{IgM}, \operatorname{IgG1-4}$, IgA1 and IgA2 against spike (Figure 2A) and RBD (Supplemental Figure 3A). Based on the titration data for total Ig (Supplemental Figure 1), plasma was tested at 1:200 dilution, while saliva was tested at 1:4 dilution. The percentage of responders for each isotype was determined using cut-off values, which were calculated as mean +3 standard deviations (SD) of the four negative specimens (Figure 2B).

All vaccinated and convalescent plasma specimens had detectable levels of total $\operatorname{Ig}$, IgM, and IgG1 against spike (Figure 2A, B). Similar results were observed for RBD-specific total Ig and IgG1 (Figure 2B, Supplemental Figure 3A), while RBD-specific plasma IgM was detected in fewer samples due to high background (Figure 2B, Supplemental Figure 3A), in agreement with our previous findings (12). Saliva total Ig and IgG1 against spike and RBD were also detected in most vaccinated and convalescent subjects. Interestingly, although anti-spike IgM was present in plasma from all vaccine recipients and convalescent patients, saliva $\operatorname{IgM}$ was detected in only a few individuals and at low levels approaching background (Figure 2A, B), providing evidence for the discordance in IgM responses in saliva versus plasma.

A significant proportion ( $>86 \%$ ) of plasma specimens from vaccinated subjects displayed IgG2, IgG3, and IgG4 Abs against spike and RBD (Figure 2B, top panels), albeit at relatively low levels compared to IgG1 (Figure 2A, top panels and Supplemental Figure 3). Low levels of these minor IgG subtypes were also detected in saliva from some vaccinees $(>43 \%)$. In the convalescent group, the percentages of IgG2-4 responders were much lower (Figure 2B, bottom panels) and the levels were near the cut-offs in plasma and saliva (Figure $2 \mathbf{A}$, bottom panels).

Of IgA subtypes, IgA1 predominated over IgA2 in both plasma and saliva samples. Among vaccinees, $100 \%$ had plasma IgA1 Abs against spike and $86 \%$ exhibited spike-specific IgA2, although IgA2 MFI values were near the cutoff (Figure 2A, B). Similar results were seen for RBDspecific IgA1 and IgA2, albeit with lower percent responders and higher cut-off values (Figure 2A, B and Supplemental Figure 3A). A comparable pattern was observed in convalescent plasma (Figure 2A, B bottom panels). The percentage of responders with specific IgA1 and IgA2 in saliva were surprisingly low. Saliva IgA1 against spike was detected in only $50 \%$ of vaccinees and $25 \%$ of convalescent patients. RBD-specific IgA1 and IgA2 against spike- and RBD were barely detected in saliva from vaccinated and convalescent subjects.

Correlation analysis of Ig isotypes in plasma versus saliva further revealed that IgG1 levels against both spike and RBD correlated strongly (Figure 2C). In contrast, no correlation was seen with $\operatorname{IgM}$ and $\operatorname{IgA} 1$, congruent with the differences noted in the percent $\operatorname{IgM}$ and $\operatorname{IgA} 1$ responders (Figure 2B). The correlation was sporadically observed for the other isotypes, but their MFI levels 
medRxiv preprint doi: https://doi.org/10.1101/2021.05.11.21256972; this version posted December 7, 2021. The copyright holder for this preprint (which was not certified by peer review) is the author/funder, who has granted medRxiv a license to display the preprint in perpetuity.

It is made available under a CC-BY-NC-NRRS-Iternational license

were near or below background (Supplemental Figure 3B, C).

In summary, $\mathrm{Ab}$ responses against spike, $\mathrm{RBD}, \mathrm{S} 1$, and $\mathrm{S} 2$ were detected in plasma and saliva from both vaccinated and convalescent individuals, while $\mathrm{Ab}$ responses to nucleoprotein were detected in plasma and saliva of the convalescent group only. The dominant spike- and RBD-specific $\mathrm{Ab}$ isotype in saliva and plasma of both vaccinated and convalescent groups was IgG1, albeit the levels varied among individuals. IgM responses were prevalent in plasma of both vaccinated and convalescent groups but were not observed in most saliva samples. Induction of IgA1 predominated over IgA 2 following vaccination and infection and was more prevalent in plasma than saliva.

\section{Plasma neutralizing activities against wild type versus B.1.351, B.1.1.7 and B.1.617.2} variants. Neutralizing activities by vaccine- and infection-induced plasma Abs were examined against WT and variant SARS-CoV-2 strains. Serially titrated specimens from seven vaccinated individuals, seven convalescent COVID-19, and three non-vaccinated COVID-19-negative controls were tested. Neutralization assays were performed using recombinant VSV (rVSV) expressing WT, B.1.1.7 (alpha), B.1.351 (beta), or B.1.617.2 (delta) spike proteins (29). The rVSV neutralization correlated strongly with live SARS-CoV-2 virus neutralization, demonstrating Spearman's r $>0.9$ across multiple studies (29). Control samples showed background neutralization below or near 50\% against all four viruses. All samples from vaccinated and convalescent groups attained $>50 \%$ neutralization against WT (Figure 3A). In fact, all achieved near or above $90 \%$ neutralization. Similar results were observed for neutralization against B.1.1.7 and B.1.617.2. In contrast, 2 vaccinee samples and 1 convalescent specimen did not reach 50\% neutralization against the B.1.351 variant. The $\mathrm{IC}_{50}$ titers against B.1.351 were also lower than the titers against WT (6-fold change in median) (Figure 3B), while the titers against B.1.1.7, B.1.617.2 and WT were not similar (Figure 3C). Of note, no difference was apparent in $\mathrm{IC}_{50}$ titers of vaccinated versus convalescent subjects against all four strains.

Detection of spike-specific ADCP activities in plasma of all vaccinated and convalescent donors. Because Ig isotypes are key determinants of Fc functions, we examined the Fc-mediated $\mathrm{Ab}$ activities from plasma specimens for both vaccinated and convalescent donors. Two Fc-dependent functions were evaluated: 1) spike-specific ADCP using THP-1 phagocytes and spike-coated fluorescent beads and 2) complement activation based on C1q and C3d deposition on spike-Ab and RBD-Ab complexes.

Spike-specific ADCP was detected above control in each of the specimens from all vaccinated and convalescent subjects (Figure 4A, B) and the levels corresponded with the spikespecific total Ig levels (Figure 4C). To assess the ADCP capacity and account for Ig level differences among samples, we calculated the AUC ratios of spike-specific ADCP over spike-binding total Ig. No difference between vaccinated and convalescent subjects was observed (Figure 4D). Of note, ADCP assays were performed with saliva samples, but no activity was detectable above background. When saliva was concentrated, ADCP was measurable in few specimens (data not shown), but the volumes of most samples were inadequate, precluding their assessment in this and other functional assays.

C1q and C3d deposition on spike- and RBD-specific Abs in plasma from vaccinated and convalescent subjects. $\mathrm{C} 1 \mathrm{q}$ and $\mathrm{C} 3 \mathrm{~d}$ deposition on antigen-Ab complexes was measured utilizing antigen-coupled bead assays. The data show variability in $\mathrm{C} 1 \mathrm{q}$ and $\mathrm{C} 3 \mathrm{~d}$ binding to spike-bound plasma Abs from both vaccinated and convalescent groups (Figure 5A, B). C1q and C3d binding levels correlated strongly (Figure 5C). Interestingly, C1q and C3d deposition was detected only in 
one convalescent plasma sample and at low levels (Figure 5A, B). In the vaccinated group, one sample did not show any $\mathrm{C} 1 \mathrm{q}$ or $\mathrm{C} 3 \mathrm{~d}$ binding to spike, and the remaining six exhibited a range of C1q and C3d binding levels above the control. Similar results were observed with C1q and C3d binding to RBD-specific plasma Abs (Supplemental Figure 4). The calculated ratios of C1q or C3d AUC to total Ig AUC further indicate higher capacity of vaccine-induced Abs to bind and activate complement (Figure 5D). These findings were supported by data from additional 20 vaccinated and 13 convalescent donors from separate cohorts, in which the greater capacity of vaccine-induced Abs to bind C1q and C3d was even more pronounced (Figure 5E and Supplemental Figures 5 and 6).

The differential complement binding activity is likely related to the relative levels of $\mathrm{IgG}$ subtypes generated by vaccination compared to infection. The IgG1 and IgG3 subtypes in particular have greater potency to activate the classical complement cascade (12). Indeed, the relative levels of IgG1+IgG3 over IgG2+IgG4 were higher in plasma from the vaccine group than the convalescent group (Figure 2). The low IgG3 levels were also observed with the larger cohort of convalescent plasma previously reported (12). To support this data, regression analyses were performed and showed that among the spike- and RBD-binding Ig isotypes tested, IgG1 and IgG3 Abs contributed most significantly to the complement binding activities $\left(\mathrm{r}^{2}=0.74-0.95, \mathrm{p}<0.0001\right)$.

The functional properties of plasma Abs induced after vaccination versus natural infection are summarized in Figure 6. The heatmap clearly shows more potent complement activation in plasma from vaccinated versus convalescent groups, even though neutralization and ADCP potencies were indistinguishable.

\section{Discussion}

This study provides evidence that plasma and saliva levels of Abs elicited after vaccination or infection correlate strongly. The data bolster previous findings showing that Abs against spike and nucleoprotein were similarly detected in plasma and saliva following SARS-CoV-2 infection (31). The total levels of Abs in saliva, however, were about 100-fold lower than in plasma. Consequently, lower percentages of responders were observed for saliva versus plasma Abs, with more notable differences for $\mathrm{S} 2$ which induces the lowest $\mathrm{Ab}$ titers among the five antigens tested. Nonetheless, saliva Abs against spike, RBD, and S1 were readily detected in the majority of vaccinated and convalescent groups, and saliva Abs against nucleoprotein were detectable in all convalescent individuals tested. While these data indicate the potential use of saliva for monitoring of anti-spike $\mathrm{Ab}$ responses in vaccinated and convalescent individuals, lower positive responses were detected, indicating the lower sensitivity of Ab detection in saliva. Differential Ig isotypes were also seen in saliva versus plasma, although the functional implications are unclear as the antiviral activities of saliva Abs have not been investigated.

Our isotyping analysis demonstrated that $\mathrm{IgG} 1$ is the dominant isotype in both plasma and saliva from all vaccinated individuals and convalescent patients. However, the IgM and IgA levels were lower in saliva versus plasma. This contrasts to recent findings in milk from convalescent mothers where the dominant spike-specific $\mathrm{Ab}$ responses were $\operatorname{IgA}$ and this response was not necessarily associated with induction of $\operatorname{IgG}$ or IgM Abs (32). However, after vaccination milk Ab responses were dominated by $\operatorname{IgG~(33).~Our~data~further~show~that~compared~to~natural~infection,~}$ vaccination induces a higher prevalence for IgG2-4 Abs both in plasma and saliva, albeit at relatively low levels. Of note, $100 \%$ of plasma from vaccinated subjects had detectable spike and RBD-specific IgG3 Abs, while only $57 \%$ and $29 \%$ responders were observed for convalescent plasma, respectively. The samples tested in this study were obtained $>189$ days post symptom onset, but the pattern was similar to that seen in convalescent plasma collected earlier ( $<8$ weeks post symptom onset, $7-17 \%$ 
responders for spike- and RBD-specific IgG3 Abs) (12), indicating that this IgG subtype profile is maintained throughout the observation period.

We examined the potential plasma neutralization against the initial Seattle WA1 strain (WT) and SARS-CoV-2 variants of concern (B.1.1.7, B.1.351, B.1.617.2), and observed potent neutralization activity against WT in each of the studied samples. In agreement with published reports $(34,35)$, weaker neutralization activities were seen against B.1.351 (beta), while neutralization of B1.1.7 (alpha) and B.1.617.2 (delta) was comparable to that of WT (36-39). No difference was seen in the $\mathrm{IC}_{50}$ titers against each of these four viruses between vaccine and convalescent groups. The neutralization titers against WT after vaccination were also similar to those of convalescent samples collected at earlier time points ( $<8$ weeks after symptom onset) (12). The effects of these mutations on the non-neutralizing Fc-dependent functions are yet to be determined. Similar to neutralization, spike-specific ADCP activities were detected in plasma from all vaccinated and convalescent individuals. However, no correlation was observed between neutralization and ADCP activities (data not shown). Moreover, complement binding activities were distinct from neutralization and ADCP, suggesting that these functions may be mediated by distinct Ig populations or by Abs targeting different epitopes. The ability to thwart neutralization may offer an advantage to the transmissibility of these and other emerging variants, but the significance of Fc-mediated antiviral activities remains unclear.

While neutralization and ADCP capacity induced by vaccination and infection were indistinguishable, vaccine-induced plasma Abs displayed a more robust ability to mediate complement binding and activation as compared to infection-induced counterparts. The differential potencies were apparent when Ab levels were considered and when comparison was made with infection-induced Abs from earlier or later time points (data not shown). Evaluation of more plasma samples independently collected from separate cohorts of vaccinated and convalescent donors revealed a consistent pattern with significantly greater capacities of vaccine-induced Abs to bind and activate complement. The mRNA vaccine-induced Ab responses were also reported in recent work with $>8000$ finger stick blood specimens to have higher seroconversion rates and greater crossreactivity with SARS-CoV-1 and Middle Eastern respiratory syndrome (MERS)-CoV RBDs (40), implying the superior quality of vaccine-induced $\mathrm{Ab}$ responses. Greater complement deposition activity was associated with higher levels of IgG1 and IgG3, the two IgG subtypes with the highest potency for complement fixation. Nonetheless, our study was limited by its relatively small sample sizes that are also restricted to mRNA vaccine recipients and sample collection at only one time point, precluding us from evaluating Abs elicited by other types of vaccines and from assessing changes of vaccine-induced responses over time. Analysis of longitudinally collected specimens with a larger sample size from recipients of different COVID-19 vaccines are warranted to reach definitive conclusions.

In addition to Ig isotypes, a parameter known to influence complement binding is Fc glycosylation, as the removal of terminal galactose from IgG Fc glycans has been shown to decrease $\mathrm{C} 1 \mathrm{q}$ binding and downstream classical complement activation without affecting Fc $\gamma \mathrm{R}$-mediated functions $(41,42)$. Similarly, the sialylation of IgG Fc domains has been demonstrated to impair complement-dependent cytotoxicity (43). The Fc glycan compositions of vaccine- and infectioninduced Abs are yet unknown. The importance of complement binding/activation for protection against SARS-CoV-2 also requires further investigation. It should be noted, however, that human neutralizing monoclonal Abs against SARS-CoV-2 requires a functional Fc region capable of binding complement and engaging Fc $\gamma \mathrm{R}$ for ADCP and ADCC, for optimal protection therapy (44). 
In conclusion, this study demonstrated that saliva and plasma Ab responses against SARS$\mathrm{CoV}-2$ antigens were elicited following vaccination or infection. Ab responses in plasma and saliva correlated significantly, although Ig isotypic differences were noted between the vaccinated and convalescent individuals. Moreover, vaccination- and infection-induced plasma Abs exhibited Fabmediated and Fc-dependent functions that included neutralization against WT and variants, phagocytosis, and complement activation. This study provide initial evidence for a superior potency of vaccine-induced Abs against spike to activate complement via the classical pathway, although the clinical significance of this function remains unclear and requires further investigation. 


\section{References}

363 1. Amanat F, Krammer F. SARS-CoV-2 Vaccines: Status Report. Immunity (2020)

364 doi:10.1016/j.immuni.2020.03.007

365 2. Le TT, Andreadakis Z, Kumar A, Román RG, Tollefsen S, Saville M, Mayhew S. The

366 COVID-19 vaccine development landscape. Nat Rev Drug Discov (2020) 19:305-306.

367 doi:10.1038/d41573-020-00073-5

$368 \quad 3 . \quad$ Yan R, Zhang Y, Li Y, Xia L, Guo Y, Zhou Q. Structural basis for the recognition of SARS-

369 CoV-2 by full-length human ACE2. Science (2020) 367:1444-1448. doi:10.1126/science.abb2762

370 4. Hoffmann M, Kleine-Weber H, Schroeder S, Krüger N, Herrler T, Erichsen S, Schiergens TS,

371 Herrler G, Wu N-H, Nitsche A, et al. SARS-CoV-2 Cell Entry Depends on ACE2 and TMPRSS2 and

372 Is Blocked by a Clinically Proven Protease Inhibitor. Cell (2020) 181:271-280.e8.

373 doi:10.1016/j.cell.2020.02.052

374 5. Walls AC, Park Y-J, Tortorici MA, Wall A, McGuire AT, Veesler D. Structure, Function, and

375 Antigenicity of the SARS-CoV-2 Spike Glycoprotein. Cell (2020) 181:281-292.e6.

376 doi:10.1016/j.cell.2020.02.058

377 6. García-Montero C, Fraile-Martínez O, Bravo C, Torres-Carranza D, Sanchez-Trujillo L,

378 Gómez-Lahoz AM, Guijarro LG, García-Honduvilla N, Asúnsolo A, Bujan J, et al. An Updated

379 Review of SARS-CoV-2 Vaccines and the Importance of Effective Vaccination Programs in

380 Pandemic Times. Vaccines (2021) 9:433. doi:10.3390/vaccines9050433

381 7. Marovich M, Mascola JR, Cohen MS. Monoclonal Antibodies for Prevention and Treatment

382 of COVID-19. JAMA (2020) 324:131-132. doi:10.1001/jama.2020.10245

383 8. Charles A Janeway J, Travers P, Walport M, Shlomchik MJ. The mucosal immune system.

384 Immunobiol Immune Syst Health Dis 5th Ed (2001) Available at:

385 https://www.ncbi.nlm.nih.gov/books/NBK27169/ [Accessed August 11, 2021]

386 9. Isho B, Abe KT, Zuo M, Jamal AJ, Rathod B, Wang JH, Li Z, Chao G, Rojas OL, Bang YM, 387 et al. Persistence of serum and saliva antibody responses to SARS-CoV-2 spike antigens in COVID-

38819 patients. Sci Immunol (2020) 5: doi:10.1126/sciimmunol.abe5511

389 10. Sterlin D, Mathian A, Miyara M, Mohr A, Anna F, Claër L, Quentric P, Fadlallah J,

390 Devilliers H, Ghillani P, et al. IgA dominates the early neutralizing antibody response to SARS-CoV-

391 2. Sci Transl Med (2021) 13:eabd2223. doi:10.1126/scitranslmed.abd2223

392 11. Cervia C, Nilsson J, Zurbuchen Y, Valaperti A, Schreiner J, Wolfensberger A, Raeber ME,

393 Adamo S, Weigang S, Emmenegger M, et al. Systemic and mucosal antibody responses specific to

394 SARS-CoV-2 during mild versus severe COVID-19. J Allergy Clin Immunol (2021) 147:545-557.e9.

395 doi:10.1016/j.jaci.2020.10.040

396 12. Klingler J, Weiss S, Itri V, Liu X, Oguntuyo KY, Stevens C, Ikegame S, Hung C-T,

397 Enyindah-Asonye G, Amanat F, et al. Role of IgM and IgA Antibodies in the Neutralization of

398 SARS-CoV-2. J Infect Dis (2020) doi:10.1093/infdis/jiaa784 
13. Rogliani P, Chetta A, Cazzola M, Calzetta L. SARS-CoV-2 Neutralizing Antibodies: A

Network Meta-Analysis across Vaccines. Vaccines (2021) 9: doi:10.3390/vaccines9030227

401

402

403

404

405

406

407

408

409

410

411

412

413

414

415

416

417

418

419

420

421

422

423

424

425

426

427

428

429

430

431

432

433

434

435

436

14. Polack FP, Thomas SJ, Kitchin N, Absalon J, Gurtman A, Lockhart S, Perez JL, Pérez Marc

G, Moreira ED, Zerbini C, et al. Safety and Efficacy of the BNT162b2 mRNA Covid-19 Vaccine. $N$ Engl J Med (2020) 383:2603-2615. doi:10.1056/NEJMoa2034577

15. Walsh EE, Frenck RW, Falsey AR, Kitchin N, Absalon J, Gurtman A, Lockhart S, Neuzil K, Mulligan MJ, Bailey R, et al. Safety and Immunogenicity of Two RNA-Based Covid-19 Vaccine Candidates. N Engl J Med (2020) 383:2439-2450. doi:10.1056/NEJMoa2027906

16. Sahin U, Muik A, Derhovanessian E, Vogler I, Kranz LM, Vormehr M, Baum A, Pascal K, Quandt J, Maurus D, et al. COVID-19 vaccine BNT162b1 elicits human antibody and TH1 T cell responses. Nature (2020) 586:594-599. doi:10.1038/s41586-020-2814-7

17. Jackson LA, Anderson EJ, Rouphael NG, Roberts PC, Makhene M, Coler RN, McCullough MP, Chappell JD, Denison MR, Stevens LJ, et al. An mRNA Vaccine against SARS-CoV-2 Preliminary Report. N Engl J Med (2020) 0:null. doi:10.1056/NEJMoa2022483

18. Tauzin A, Nayrac M, Benlarbi M, Gong SY, Gasser R, Beaudoin-Bussières G, Brassard N, Laumaea A, Vézina D, Prévost J, et al. A single dose of the SARS-CoV-2 vaccine BNT162b2 elicits Fc-mediated antibody effector functions and T cell responses. Cell Host Microbe (2021) 29:11371150.e6. doi:10.1016/j.chom.2021.06.001

19. Bournazos S, Ravetch JV. Diversification of IgG effector functions. Int Immunol (2017) 29:303-310. doi:10.1093/intimm/dxx025

20. Goldberg BS, Ackerman ME. Antibody-mediated complement activation in pathology and protection. Immunol Cell Biol (2020) 98:305-317. doi:10.1111/imcb.12324

21. Pincetic A, Bournazos S, DiLillo DJ, Maamary J, Wang TT, Dahan R, Fiebiger B-M, Ravetch JV. Type I and type II Fc receptors regulate innate and adaptive immunity. Nat Immunol (2014) 15:707-716. doi:10.1038/ni.2939

22. Kellner C, Otte A, Cappuzzello E, Klausz K, Peipp M. Modulating Cytotoxic Effector Functions by Fc Engineering to Improve Cancer Therapy. Transfus Med Hemotherapy Off Organ Dtsch Ges Transfusionsmedizin Immunhamatologie (2017) 44:327-336. doi:10.1159/000479980

23. Lee C-H, Romain G, Yan W, Watanabe M, Charab W, Todorova B, Lee J, Triplett K, Donkor $\mathrm{M}$, Lungu OI, et al. IgG Fc domains that bind C1q but not effector Fc $\gamma$ receptors delineate the importance of complement-mediated effector functions. Nat Immunol (2017) 18:889-898. doi:10.1038/ni.3770

24. Weiss S, Klingler J, Hioe C, Amanat F, Baine I, Arinsburg S, Kojic EM, Stoever J, Liu STH, Jurczyszak D, et al. A High Through-Put Assay For Circulating Antibodies Directed Against The S Protein Of Severe Acute Respiratory Syndrome Coronavirus 2 (Sars-Cov-2). J Infect Dis (2020) doi:10.1093/infdis/jiaa531

25. Amanat F, Stadlbauer D, Strohmeier S, Nguyen THO, Chromikova V, McMahon M, Jiang K, Arunkumar GA, Jurczyszak D, Polanco J, et al. A serological assay to detect SARS-CoV-2

This is a provisional file, not the final typeset article 
seroconversion in humans. Nat Med (2020)1-4. doi:10.1038/s41591-020-0913-5

26. Stadlbauer D, Amanat F, Chromikova V, Jiang K, Strohmeier S, Arunkumar GA, Tan J,

27. Perez LG, Martinez DR, deCamp AC, Pinter A, Berman PW, Francis D, Sinangil F, Lee C,

28. Oguntuyo KY, Stevens CS, Hung C-T, Ikegame S, Acklin JA, Kowdle SS, Carmichael JC,

29. Ikegame S, Siddiquey MNA, Hung C-T, Haas G, Brambilla L, Oguntuyo KY, Kowdle S,

SARS-CoV-2 variants. Nat Commun (2021) 12:4598. doi:10.1038/s41467-021-24909-9

30. Balasubramanian P, Williams C, Shapiro MB, Sinangil F, Higgins K, Nádas A, Totrov M, and V3 of HIV gp120 in the VAX003 and VAX004 Vaccine Trials. Sci Rep (2018) 8:542. doi:10.1038/s41598-017-18863-0 SARS-CoV-2-Specific Antibody Responses in Saliva. J Clin Microbiol (2020) 59: doi:10.1128/JCM.02204-20 23:101735. doi:10.1016/j.isci.2020.101735

33. Fox A, Norris C, Amanat F, Zolla-Pazner S, Powell RL. The vaccine-elicited SARS-CoV-2 Variants of Concern in Hospitalized COVID-19 Patients. Viruses (2021) 13:1347. doi:10.3390/v13071347 Lancet Microbe (2021) 2:e283-e284. doi:10.1016/S2666-5247(21)00068-9

473 36. Bian L, Gao F, Zhang J, He Q, Mao Q, Xu M, Liang Z. Effects of SARS-CoV-2 variants on 
37. Bates TA, Leier HC, Lyski ZL, McBride SK, Coulter FJ, Weinstein JB, Goodman JR, Lu Z,

479 38. Carreño JM, Alshammary H, Singh G, Raskin A, Amanat F, Amoako A, Gonzalez-Reiche 480 AS, van de Guchte A, Study Group P, Srivastava K, et al. Evidence for retained spike-binding and 481 neutralizing activity against emerging SARS-CoV-2 variants in serum of COVID-19 mRNA vaccine recipients. EBioMedicine (2021) 73:103626. doi:10.1016/j.ebiom.2021.103626

483 39. Dupont L, Snell LB, Graham C, Seow J, Merrick B, Lechmere T, Maguire TJA, Hallett SR, infection in humans with SARS-CoV-2 and variants of concern. Nat Microbiol (2021) doi:10.1038/s41564-021-00974-0 Grabar C, et al. Substantial Differences in SARS-CoV-2 Antibody Responses Elicited by Natural Infection and mRNA Vaccination. bioRxiv (2021)2021.04.15.440089.

490 doi:10.1101/2021.04.15.440089

491 41. Hodoniczky J, Zheng YZ, James DC. Control of recombinant monoclonal antibody effector functions by Fc N-glycan remodeling in vitro. Biotechnol Prog (2005) 21:1644-1652. doi:10.1021/bp050228w

42. Boyd PN, Lines AC, Patel AK. The effect of the removal of sialic acid, galactose and total carbohydrate on the functional activity of Campath-1H. Mol Immunol (1995) 32:1311-1318. doi:10.1016/0161-5890(95)00118-2

43. Quast I, Keller CW, Maurer MA, Giddens JP, Tackenberg B, Wang L-X, Münz C, Nimmerjahn F, Dalakas MC, Lünemann JD. Sialylation of IgG Fc domain impairs complementdependent cytotoxicity. J Clin Invest 125:4160-4170. doi:10.1172/JCI82695

500 44. Winkler ES, Gilchuk P, Yu J, Bailey AL, Chen RE, Chong Z, Zost SJ, Jang H, Huang Y, 501 Allen JD, et al. Human neutralizing antibodies against SARS-CoV-2 require intact Fc effector 502 functions for optimal therapeutic protection. Cell (2021) 184:1804-1820.e16.

503 doi:10.1016/j.cell.2021.02.026 


\section{Conflict of interest}

507 The Icahn School of Medicine at Mount Sinai has filed patent applications relating to SARS-CoV-2

508 serological assays and listed Dr. Viviana Simon as co-inventor. Mount Sinai has spun out a company,

509 Kantaro, to market serological tests for SARS-CoV-2. The other authors declare that the research was

510 conducted in the absence of any commercial or financial relationships that could be construed as a

511 potential conflict of interest.

\section{Author contributions}

513 J.K. and C.E.H. wrote the manuscript. J.K., G.S.L., S.Z-P, C.U., and C.E.H. designed the

514 experiments. J.K., G.S.L., V.I., and X.L. performed the experiments and collected the data. J.K.,

515 G.S.L., B.L., S.Z-P., C.U., and C.E.H. analyzed the data. H-P.C., C-T.H., S.K., F.A., and B.L.

516 provided protocols, antigens, cells and virus stocks. G.E-A., J.C.B., and S.L. obtained specimens.

517 V.S., C.R.G. and G.K. provided specimens. All authors read and approved the final manuscript.

\section{Funding}

519 This work was supported in part by the Department of Medicine of the Icahn School of Medicine at

520 Mount Sinai Department of Medicine (to S.Z-P., C.E.H.); the Department of Microbiology and the

521 Ward-Coleman estate for endowing the Ward-Coleman Chairs at the Icahn School of Medicine at

522 Mount Sinai (to B.L.), the Department of Veterans Affairs [Merit Review Grant I01BX003860] (to

523 C.E.H.) and [Research Career Scientist Award 1IK6BX004607] (to C.E.H.); the National Institutes

524

525

526

527

528

529 of Health [grant AI139290] to C.E.H., [grants R01 AI123449, R21 AI1498033] to B.L, [grant R01 AI140909] to C.U.; the NIAID Collaborative Influenza Vaccine Innovation Centers (CIVIC) [contract 75N93019C00051], NIAID Center of Excellence for Influenza Research and Surveillance (CEIRS) [contracts HHSN272201400008C and HHSN272201400006C], NIAID [grants U01AI141990 and U01AI150747], the generous support of the JPB Foundation and the Open Philanthropy Project [research grant 2020-215611[5384]] and anonymous donors to V.S.

\section{Acknowledgments}

We thank Dr. Florian Krammer for providing spike and RBD antigens, and all the donors for their contribution to the research. We would like to thank the expertise and assistance of Dr. Christopher (Hala Alshammary, Angela A Amoako, Dalles Andre, Mahmoud H Awawda, Katherine F Beach, Maria C Bermúdez-González, Juan Manuel Carreno, Gianna Cai, Rachel L Chernet, Christian Cognigni, Karine David, Lily Q Eaker, Emily D Ferreri, Daniel L Floda, Hisaaki Kawabata, Florian Krammer, Giulio Kleiner, Neko Lyttle, Wanni A Mendez, Lubbertus C F Mulder, Ismail Nabeel, Annika Oostenink, Ariel Raskin, Aria Rooker, Kayla T Russo, Ashley Beathrese T Salimbangon, Miti Saksena, Amber S Shin, Gagandeep Singh, Viviana Simon, Levy A Sominsky, Komal Srivastava, Johnston Tcheou, Ania Wajnberg) and the 
medRxiv preprint doi: https://doi.org/10.1101/2021.05.11.21256972; this version posted December 7, 2021. The copyright holder for this preprint (which was not certified by peer review) is the author/funder, who has granted medRxiv a license to display the preprint in perpetuity.

It is made available under a CC-BY-NCSNRSIIternational licerse

\section{Figure legends}

546 Figure 1. Levels of SARS-CoV-2-specific total Ig in plasma and saliva. (A) Titers of antigenspecific total Ig in plasma versus saliva specimens from vaccinated donors and convalescent COVID19 patients. End-point titers were calculated from reciprocal dilutions that reached the the cut-off values (mean $+3 \mathrm{SD}$ of negative controls at the lowest dilution). Data points below the cut-off are shown at the lowest reciprocal dilutions (100 for plasma, 2 for saliva) as gray circles (vaccinated) or gray diamonds (convalescent). (B) The percentages of responders above cut-off for each antigen based on plasma versus saliva total Ig from seven vaccinated subjects (left panel) and seven convalescent COVID-19 patients (right panel). (C) Spearman correlation of spike- and S1-specific total Ig levels in plasma versus saliva from vaccinated and convalescent subjects. Areas under the curves (AUC) were calculated from the titration curves in Supplemental Figure 1. The dotted line indicates the cut-off value.

Figure 2. Ig isotypes against SARS-CoV-2 spike and RBD in plasma versus saliva after vaccination and infection. (A) Total $\mathrm{Ig}$, IgM, IgG1, IgG2, IgG3, IgG4, IgA1 and IgA2 levels against spike were measured in plasma (left) and saliva (right) specimens from vaccinated (top panels) and convalescent COVID-19 patients (lower panels). For controls, samples from four COVID-19negative individuals (open symbols) were tested in parallel. The dotted line represents the cut-off calculated as mean of the four control specimens $+3 \mathrm{SD}$ for each isotype. (B) The percentages of responders among vaccinated (top panels) and convalescent subjects (lower panels) for each spike- or RBD-specific Ig isotype on the basis of plasma (left) and saliva (right) reactivity. (C) Spearman correlation between spike- and RBD-specific IgG1, IgA1 and IgM levels in plasma versus saliva from vaccinated and convalescent subjects.

Figure 3. Plasma neutralization activities against WT versus variants. (A) Neutralization of recombinant VSV viruses bearing the spike proteins of SARS-CoV-2 WT, B.1.531, B.1.1.7 or B.1.617.2 by plasma specimens from vaccinated and convalescent COVID-19 donors. Plasma samples from three COVID-19-negative individuals were tested in parallel; these negative control data are shown as mean + SD of replicates from all three samples. The dotted line indicates $50 \%$ neutralization. (B) Comparison of neutralization IC50 titers against WT versus B.1.351, B.1.1.7 and B.1.617.2. The specimens that did not reach $50 \%$ neutralization were shown as gray symbols at the lowest reciprocal dilution. Statistical analysis was performed using a Kruskal-Wallis test. *: p <0.05; ns: non-significant.

Figure 4. ADCP activities in plasma of vaccinated and convalescent individuals. Spike-specific ADCP activities in plasma specimens from (A) vaccinated and (B) convalescent donors were tested along with two control plasma samples from COVID-19-negative individuals. ADCP was measured by flow cytometry after incubation of plasma-treated spike-coated fluorescent beads with THP-1 phagocytes. ADCP scores were calculated as $\%$ bead $^{+}$cells $\times$MFI of bead ${ }^{+}$cells. The dotted line indicates the background. (C) Correlation between spike-specific ADCP AUC and spike-specific total Ig AUC from the seven vaccinated individuals, seven convalescent patients and two negative controls. (D) Ratio of spike-specific ADCP AUC to spike-specific total Ig AUC from the seven vaccinated individuals and seven convalescent patients. individuals. (A-B) The binding of C1q (A) and C3d (B) to spike-specific Abs in plasma specimens from vaccinated (left) and convalescent (right) donors was assessed together with four COVID-19negative controls in multiplex bead assays. Specimens were diluted 2-fold from 1:100 to 1:6,400 or 

four control specimens. $\Delta \mathrm{C} 1 \mathrm{q}$ and $\Delta \mathrm{C} 3 \mathrm{~d}$ MFI values were calculated by subtracting background MFI from each assay. (C) Spearman correlation between C1q AUC and C3d AUC values for spike- or RBD-specific Abs in plasma specimens from vaccinated and convalescent donors. (D-E) Ratio of C1q and C3d binding AUC to total Ig AUC of specimens from 7 vaccinated and 7 convalescent donors (D) and from additional 20 recipients of Pfizer or Moderna mRNA vaccines and 13 convalescent donors (E). Statistical significance was assessed using a Kruskal-Wallis test $(*: \mathrm{p}<0.05$, $* *: \mathrm{p}<0.01, * * *: \mathrm{p}<0.001, * * * *: \mathrm{p}<0.0001)$.

597 Figure 6. Heatmap to show the relative levels of neutralization $\left(\mathrm{IC}_{50}\right)$ and $\mathrm{Fc}$-mediated activities (ratios to total Ig) in plasma specimens from vaccinated and convalescent donors. Z-scores calculated for each $\mathrm{Ab}$ activity were used to generate the heatmap.

\section{Supplementary figure legends}

601 Supplemental Figure 1. Titration curves are shown for total Ig against spike, RBD, S1, S2,

602

603 nucleoprotein $(\mathrm{N})$, and BSA in plasma and saliva specimens from seven vaccinated subjects (left

604 panels), seven convalescent COVID-19 patients (right panels) and four COVID-19-negative subjects (gray). Specimens were diluted at 4-fold dilutions from 1:100 to 1:6,400 or 102,400 (plasma) or 2fold from 1:2 to 1:16 (saliva). The dotted lines indicated the cut-off values calculated as mean $+3 \mathrm{SD}$ of 1:100 diluted plasma or 1:4 diluted saliva of the four COVID-19-negative specimens. Data were generated from the multiplex bead antibody binding assay and mean fluorescent intensity (MFI) values were plotted.

609 Supplemental Figure 2. Spearman correlation of spike- versus RBD-, S1-, S2- or nucleoprotein610 specific total Ig levels in (A) plasma or (B) saliva from vaccinated and convalescent subjects.

611 Supplemental Figure 3. (A) Total Ig, IgM, IgG1, IgG2, IgG3, IgG4, IgA1 and IgA2 levels against RBD were measured in plasma (left) and saliva (right) specimens from vaccinated (top panel), convalescent COVID-19 patients (lower panel) and COVID-19-negative controls. The dotted line represents the cut-off values calculated as mean of the four control specimens $+3 \mathrm{SD}$ for each isotype. (B-C) Spearman correlation of (B) spike- and (C) RBD-specific isotypes levels in plasma versus saliva from vaccinated and convalescent subjects. The dotted line represents the cut-off.

Supplemental Figure 4. (A-B) C1q or C3d binding to RBD-specific Abs in plasma specimens from seven vaccinated individuals (left) and seven convalescent COVID-19 patients (right) and four COVID-19-negative controls. Specimens were diluted at 2-fold dilutions from 1:100 to 1:6,400 or 12,800 . The dotted line represents the 100x dilution cut-off calculated as mean of the four control specimens $+3 \mathrm{SD} . \Delta \mathrm{C} 1 \mathrm{q}(\mathrm{A})$ and $\Delta \mathrm{C} 3 \mathrm{~d}(\mathrm{~B}) \mathrm{MFI}$ values were calculated by subtracting background MFI from each assay.

623

Supplemental Figure 5. Titration curves are shown for total Ig against spike, RBD, S1, S2 and nucleoprotein in sera and plasma specimens from 20 vaccinated subjects (left panels), 13 convalescent COVID-19 patients (right panels) and four COVID-19-negative subjects (gray). Specimens were diluted at 4-fold dilutions from 1:100 to 1:12,800. The dotted lines indicated the cutoff values calculated as mean +3SD of 1:100 diluted plasma of the four COVID-19-negative specimens. Data were generated using the multiplex bead antibody binding assay and mean 
medRxiv preprint doi: https://doi.org/10.1101/2021.05.11.21256972; this version posted December 7, 2021. The copyright holder for this preprint

(which was not certified by peer review) is the author/funder, who has granted medRxiv a license to display the preprint in perpetuity.

It is made available under a CC-BY-NC-NR $\mathbf{R}$ International license 2 antibodies in plasma and saliva

630 Supplemental Figure 6. (A-B) C1q or C3d binding to spike- or RBD-specific Abs in plasma

631 specimens from 20 vaccinated individuals (left) and 13 convalescent COVID-19 patients (right) and

632 four COVID-19-negative controls. Specimens were diluted at 2-fold dilutions from 1:100 to 1:6,400

633 or 12,800. The dotted line represents the 100x dilution cut-off calculated as mean of the four control

634 specimens $+3 \mathrm{SD} . \Delta \mathrm{C} 1 \mathrm{q}(\mathrm{A})$ and $\triangle \mathrm{C} 3 \mathrm{~d}(\mathrm{~B}) \mathrm{MFI}$ values were calculated by subtracting background

635 MFI from each assay. 
Figure 1. Levels of SARS-CoV-2-specific total Ig in plasma and saliva. (A) Titers of antigen-specific total Ig in plasma versus saliva specimens from vaccinated donors and convalescent COVID-19 patients. End-point titers were calculated from reciprocal dilutions that reached the the cut off yalues (mean 3 3SD of negative controls at the lowest dilution). (which was not certified by peer review) is the author/funder, who has granted medRxiv a license to display the preprint in perpetuity. It is made available under a CC-BY-NC-ND 4.0 International license.

Data points below the cut-off are shown at the lowest reciprocal dilutions (100 for plasma, 2 for saliva) as gray circles (vaccinated) or gray diamonds (convalescent). (B) The percentages of responders above cut-off for each antigen based on plasma versus saliva total Ig from seven vaccinated subjects (left panel) and seven convalescent COVID-19 patients (right panel). (C) Spearman correlation of spike- and S1-specific total Ig levels in plasma versus saliva from vaccinated and convalescent subjects. Areas under the curves (AUC) were calculated from the titration curves in Supplemental Figure 1. The dotted line indicates the cut-off value. 

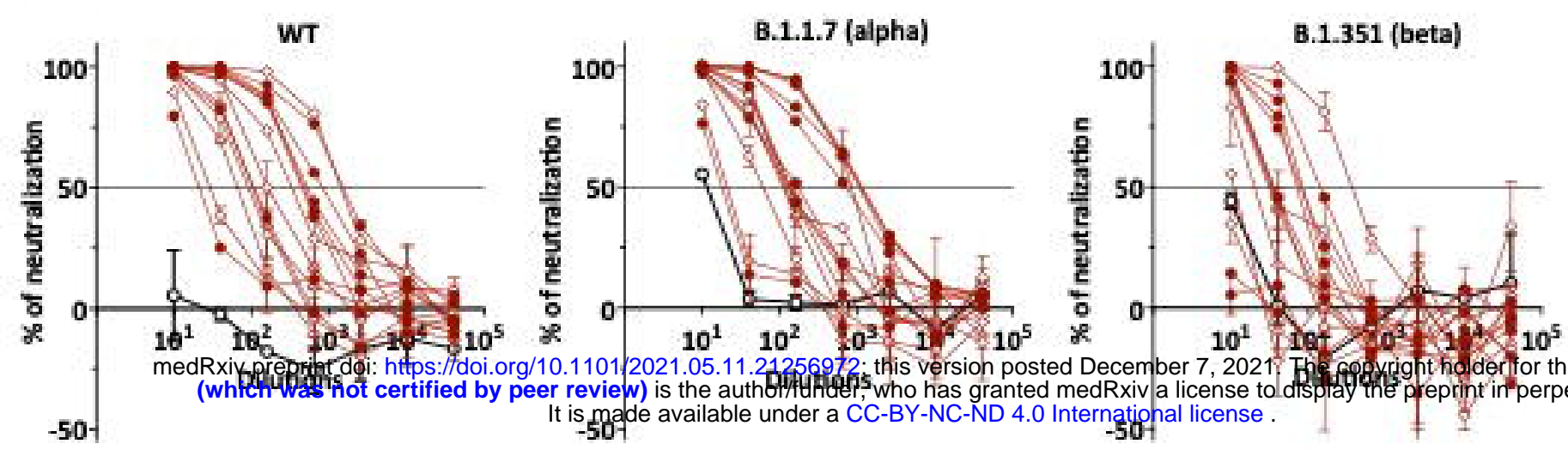

It is made available under a CC-BY-NC-ND 4.0 International license

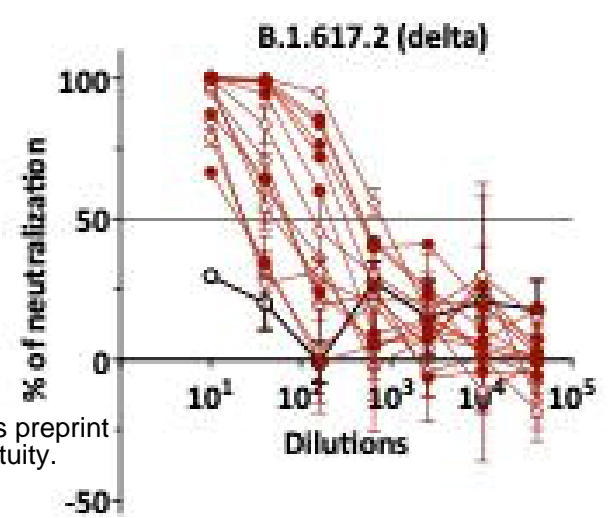

$\checkmark$ Convalescent COVID-19 patients

Vaccinated subjects

O Negative control

(B)

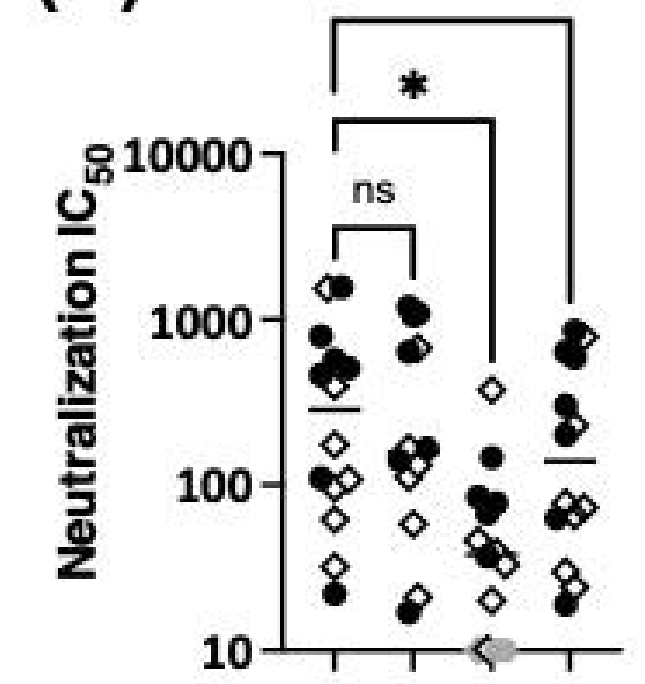

2.

$0^{2} x^{2} 0^{2}$ 
of recombinant VSV viruses bearing the spike proteins of SARS-CoV-2 WT, B.1.531,

B.1.1.7 or B.1.617.2 by plasma specimens from vaccinated and convalescent COVID-19

donors Plasma samples from three COVVID-19-negative individuals were tested in parallel; (which was not certified by peer review) is the author/funder, who has granted medRxiv a license to display the preprint in perpetuity. It is made available under a CC-BY-NC-ND 4.0 International license.

these negative control data are shown as mean $+\mathrm{SD}$ of replicates from all three samples. The dotted line indicates $50 \%$ neutralization. (B) Comparison of neutralization IC50 titers against WT versus B.1.351, B.1.1.7 and B.1.617.2. The specimens that did not reach $50 \%$ neutralization were shown as gray symbols at the lowest reciprocal dilution. Statistical analysis was performed using a Kruskal-Wallis test. ${ }^{*}$ : $\mathrm{p}<0.05$; ns: non-significant. 


\section{(A)}

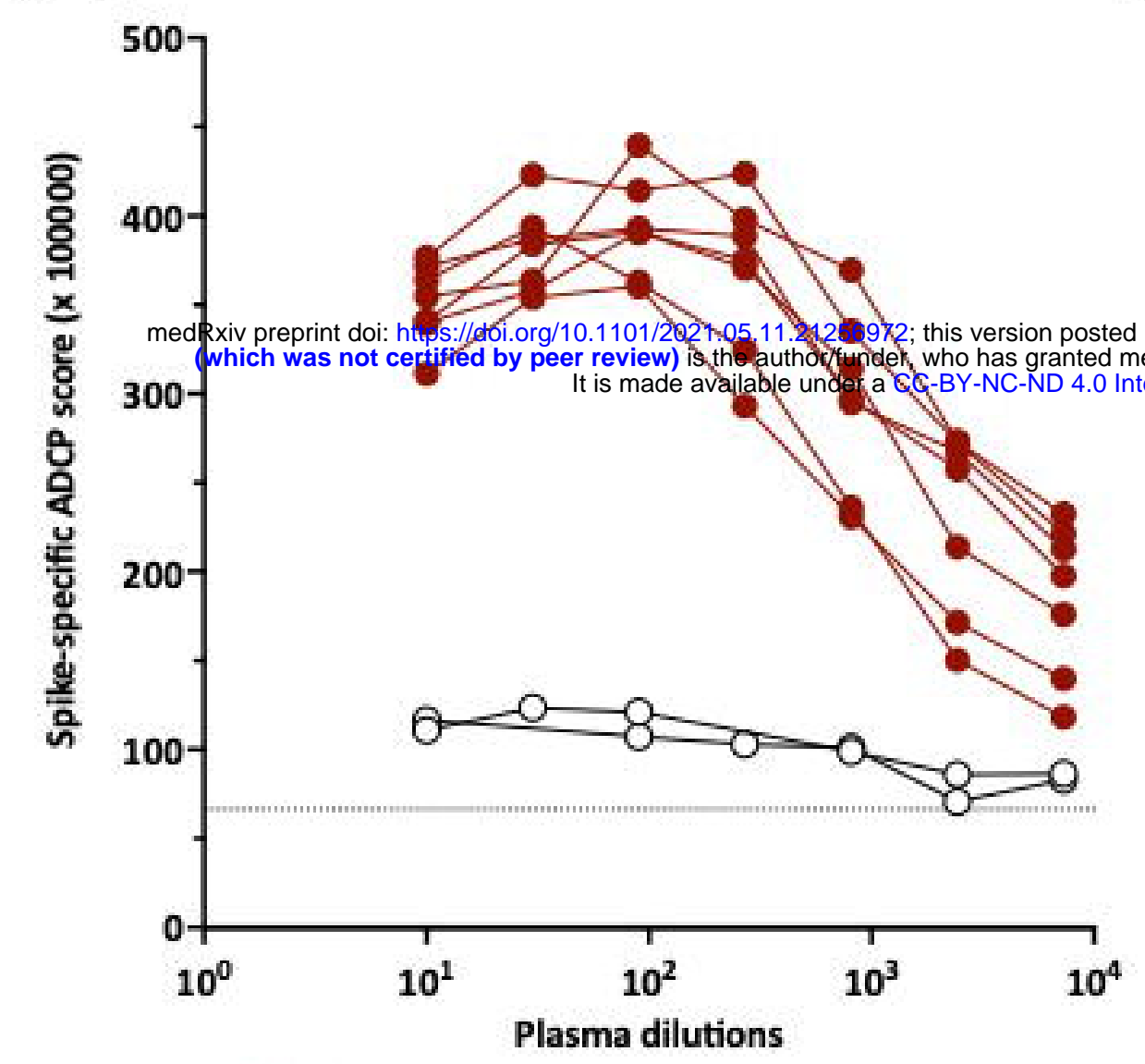

(B)

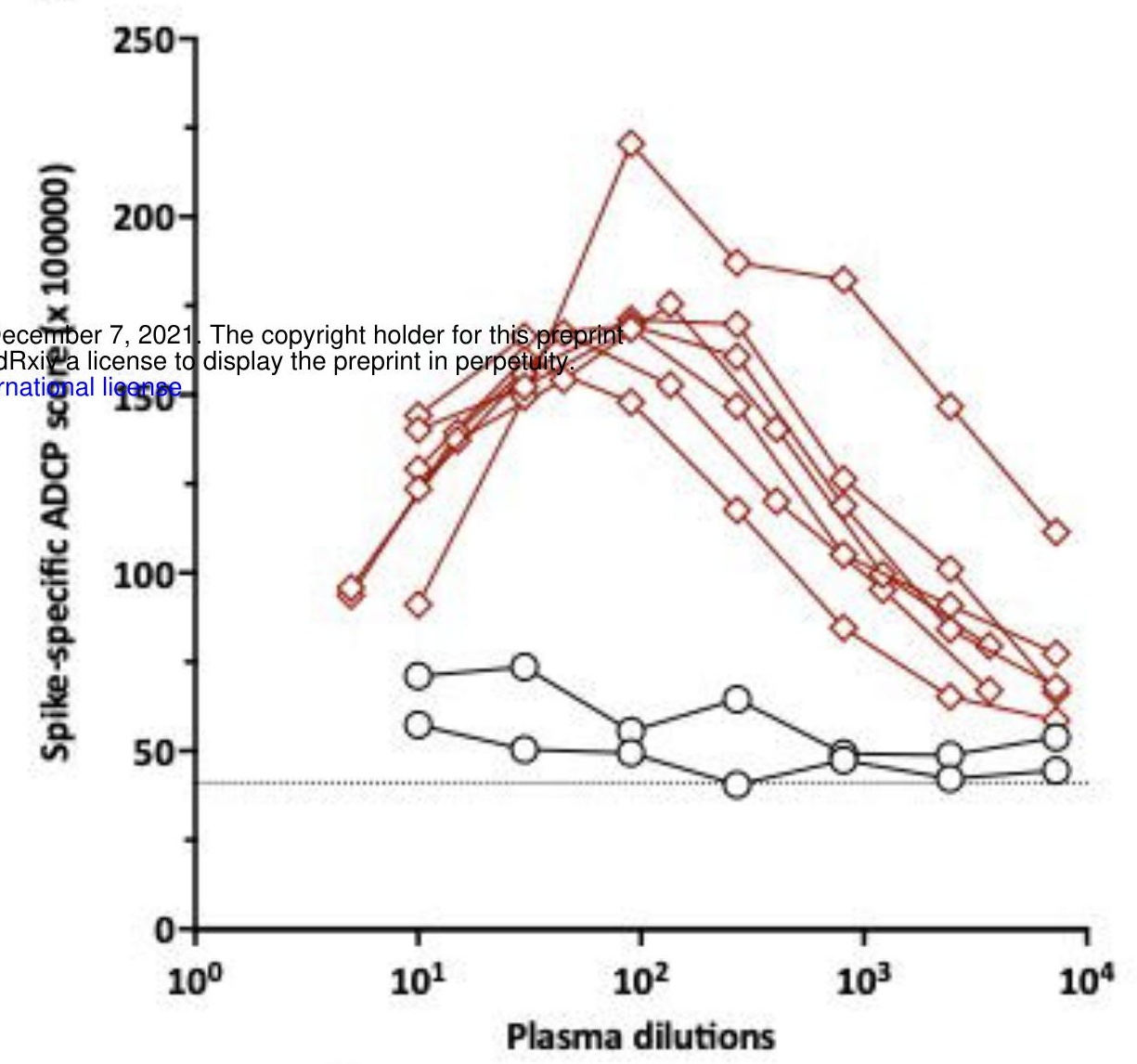

Vaccinated subjects $\mathrm{O}$ covID-19-negative subjects

$\diamond$ Convalescent COVID-19 patients
(C)

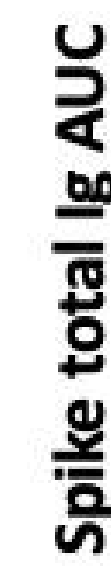

(D)

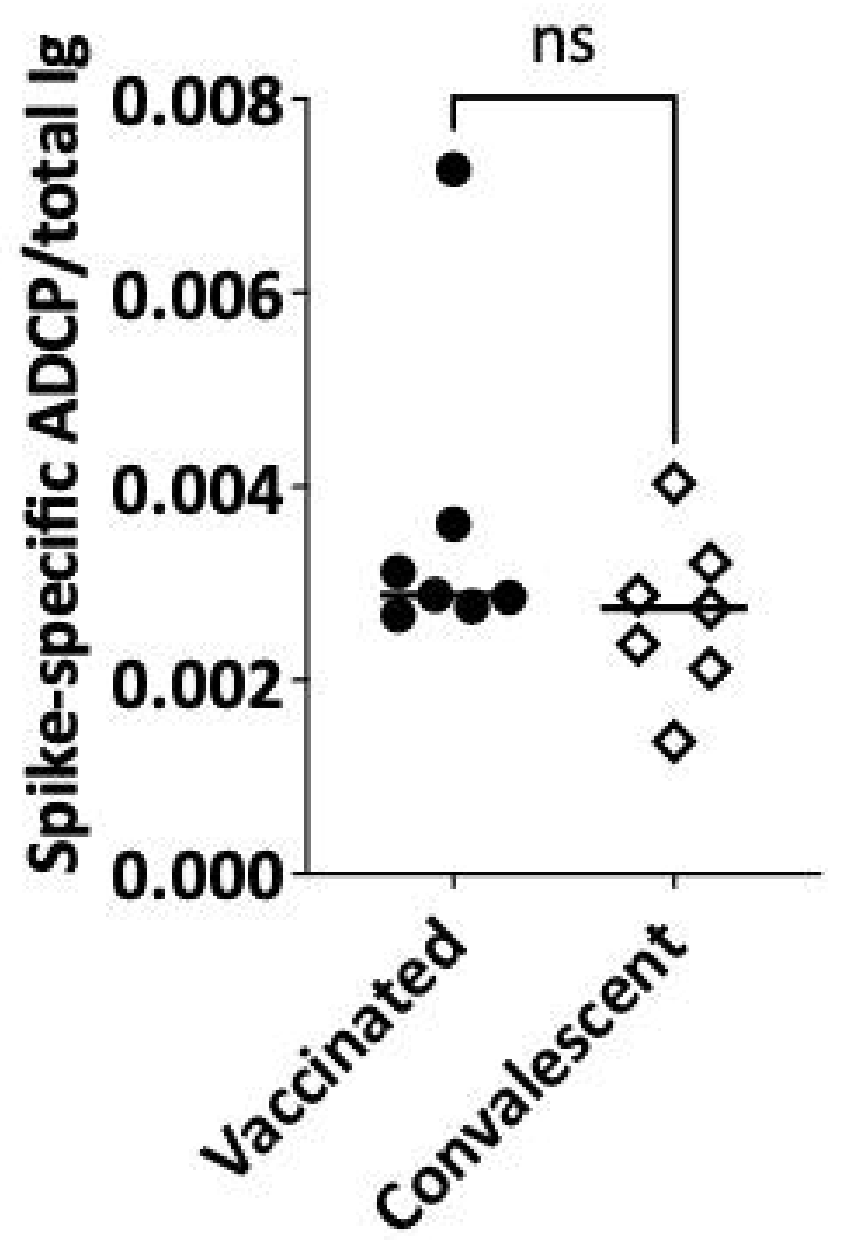


Figure 4. ADCP activities in plasma of vaccinated and convalescent individuals. Spike-

specific ADCP activities in plasma specimens from (A) vaccinated and (B) convalescent donors were tested along with two control plasma samples from COVID-19-negative individuals $\mathrm{ADCP}$ was measured by flow cytometry after incubation of plasma-treated spike(which was not certified by peer review) is the author/funder, who has granted medRxiv a license to display the preprint in perpetuity. It is made available under a CC-BY-NC-ND 4.0 International license.

coated fluorescent beads with THP-1 phagocytes. ADCP scores were calculated as \% bead + cells $\times$ MFI of bead + cells. The dotted line indicates the background. (C) Correlation between spike-specific ADCP AUC and spike-specific total Ig AUC from the seven vaccinated individuals, seven convalescent patients and two negative controls. (D) Ratio of spikespecific ADCP AUC to spike-specific total Ig AUC from the seven vaccinated individuals and seven convalescent patients. 
(A)

Spike - vaccinated

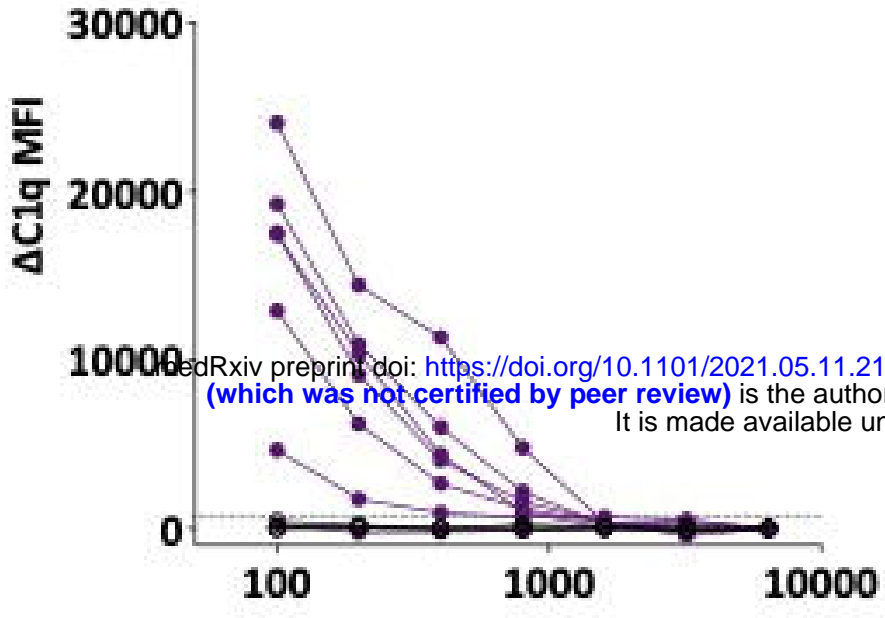

Plasma dilution

(B)

Spike - vaccinated

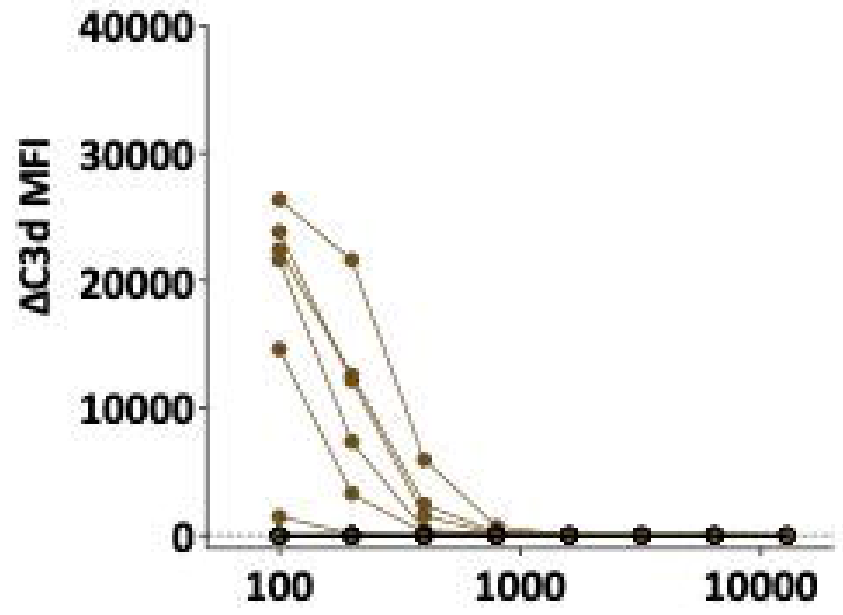

Plasma dilution
Spike - convalescent

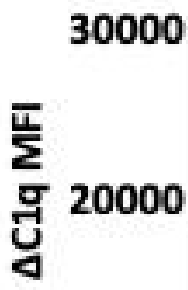

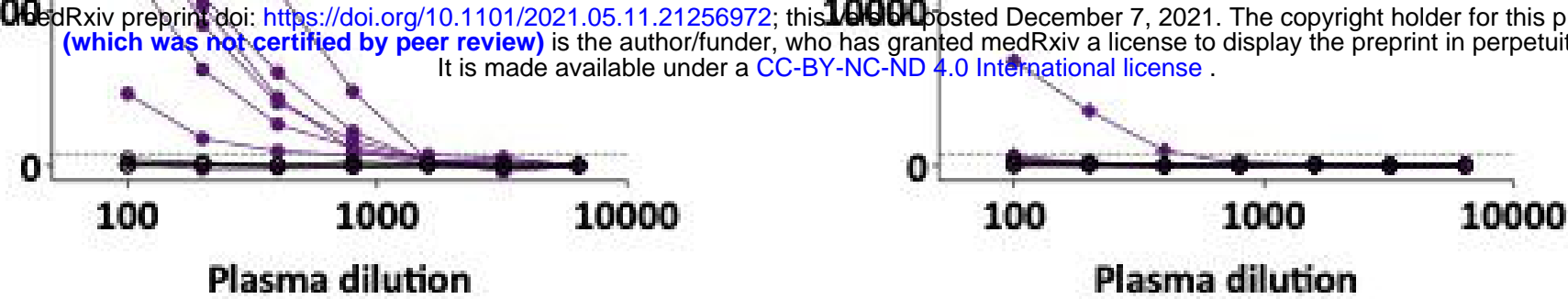

Spike - convalescent

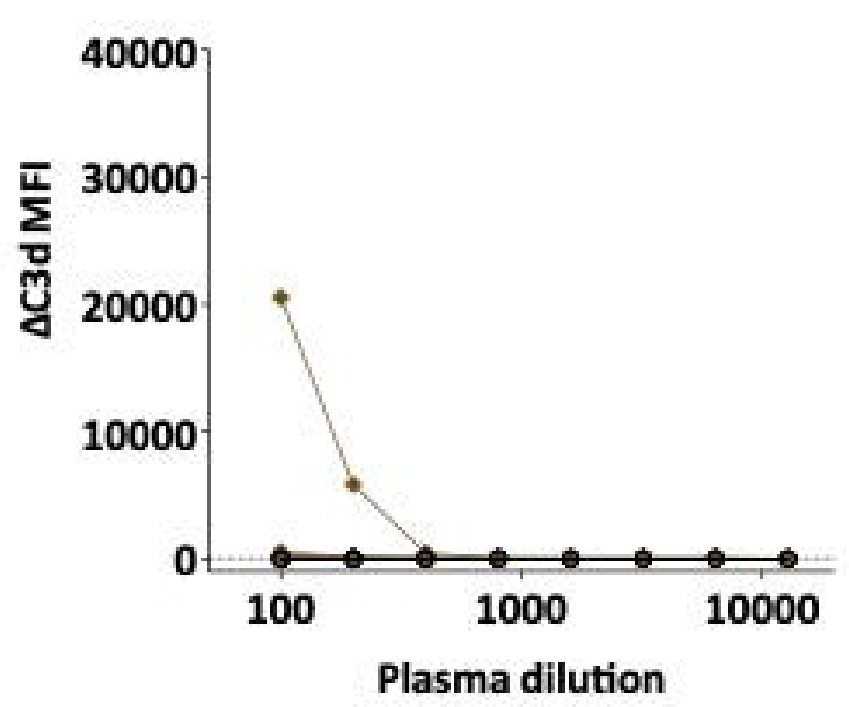

COVID-19-negative subjects

(C)
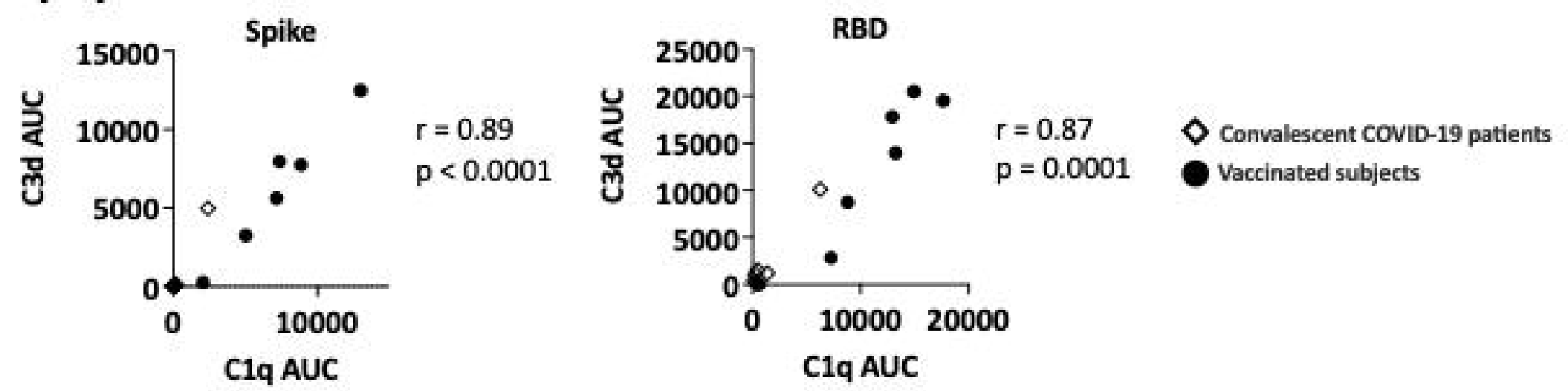

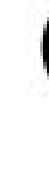

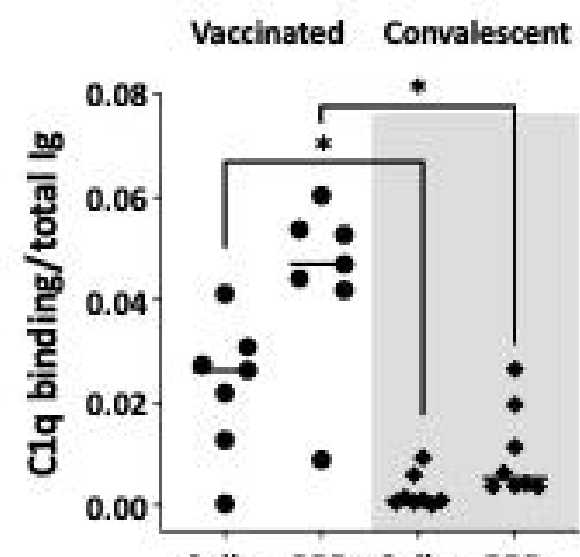

Spike RBD Spike RBD

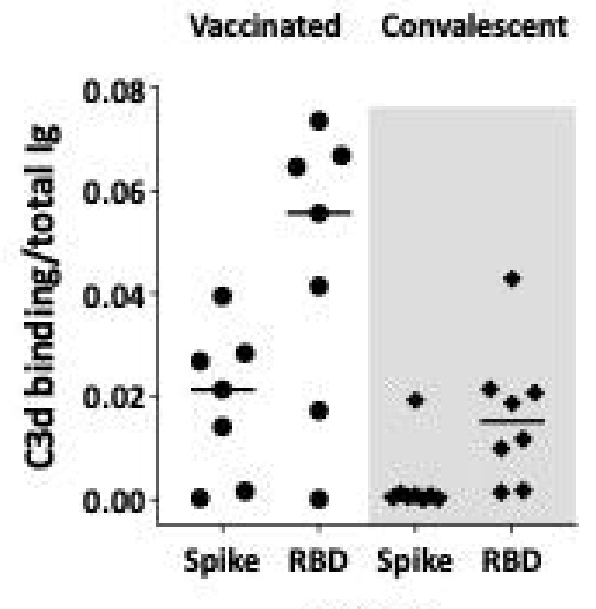

Antigen
(E)
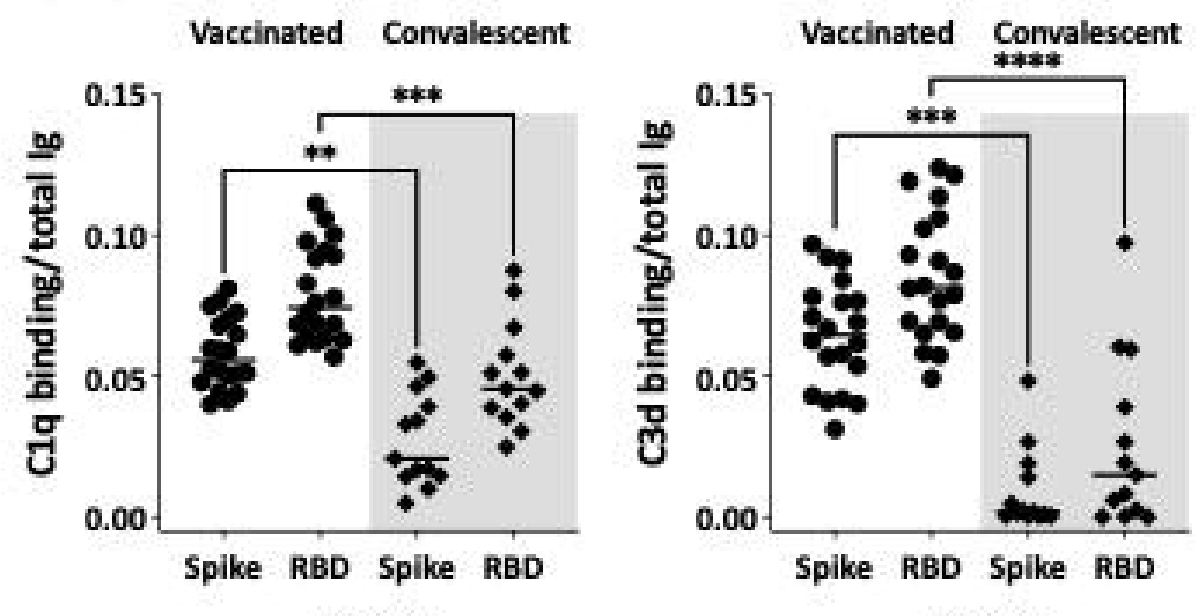
Antigen 
Figure 5. Complement-binding activities in the plasma of vaccinated and convalescent individuals. (A-B) The binding of $\mathrm{C} 1 \mathrm{q}(\mathrm{A})$ and $\mathrm{C} 3 \mathrm{~d}(\mathrm{~B})$ to spike-specific Abs in plasma specimens from vaccinated (left) and convalescent (right) donors was assessed together with four COXVID-19-megative controls in multiplex bead assays Specimens were diluted 2-fold (which was not certified by peer review) is the author/funder, who has granted medRxiv a license to display the preprint in perpetuity.

from 1:100 to $1: 6,400$ or 12,800 . The dotted line represents the $100 \mathrm{x}$ dilution cut-off values calculated as mean $+3 \mathrm{SD}$ of the four control specimens. $\Delta \mathrm{Clq}$ and $\Delta \mathrm{C} 3 \mathrm{~d}$ MFI values were calculated by subtracting background MFI from each assay. (C) Spearman correlation between C1q AUC and C3d AUC values for spike- or RBD-specific Abs in plasma specimens from vaccinated and convalescent donors. (D-E) Ratio of C1q and C3d binding AUC to total Ig AUC of specimens from 7 vaccinated and 7 convalescent donors (D) and from additional 20 recipients of Pfizer or Moderna mRNA vaccines and 13 convalescent donors (E). Statistical significance was assessed using a Kruskal-Wallis test $(*$ : $\mathrm{p}<0.05$, **: $\left.\mathrm{p}<0.01,{ }^{* * *}: \mathrm{p}<0.001,{ }^{* * * *}: \mathrm{p}<0.0001\right)$ 


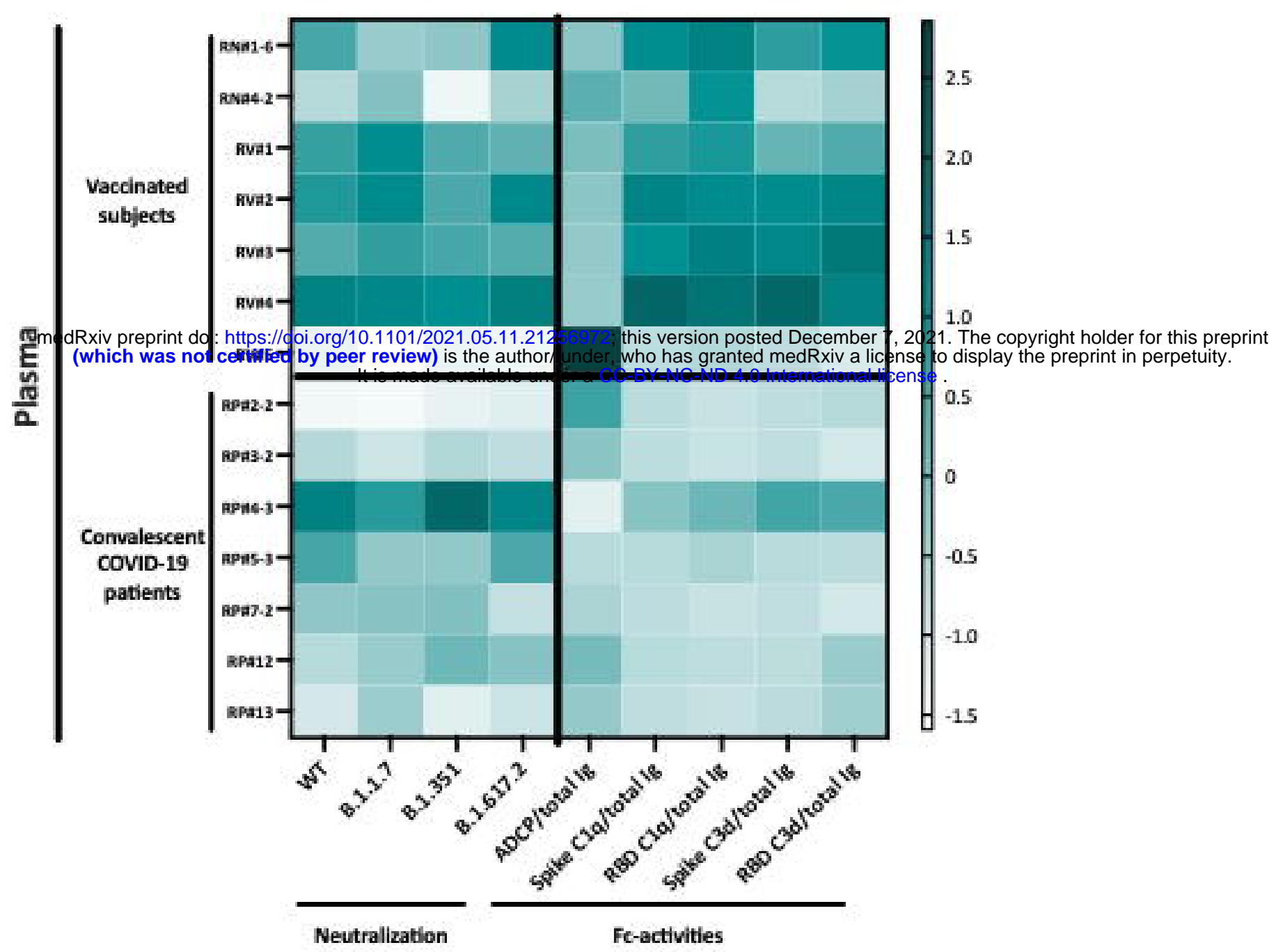

Figure 6. Heatmap to show the relative levels of neutralization (IC50) and Fc-mediated activities (ratios to total Ig) in plasma specimens from vaccinated and convalescent donors.

$\mathrm{Z}$-scores calculated for each $\mathrm{Ab}$ activity were used to generate the heatmap. 